\title{
Deal making analysis: Microsoft acquired Nokia
}

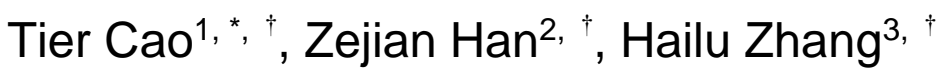 \\ ${ }^{1}$ Beijing Royal School, Beijing, China \\ 2 Haidian Foreign Language Academy, Beijing, China \\ ${ }^{3}$ Camdn Catholic high school, USA \\ *Corresponding author: 489573141@qq.com \\ These authors contributed equally.
}

\begin{abstract}
These instructions give you guidelines for preparing papers for Deal making analysis: Microsoft acquired Nokia. Microsoft made the surprising announcement that it was purchasing Finnish mobile handset maker Nokia for $\$ 7.2$ billion, a merger aimed at building Microsoft's mobile and smartphone offerings. The merger faced even more complexity after the ink dried on the contract-namely, the challenges of integrating employees from different cultures. International business negotiation case studies such as this one underscore the difficulties that companies face when attempting to negotiate two different identities.
\end{abstract}

Keywords: Microsoft, Nokia, smartphone, employee's arrangement

\section{Introduction}

Recently, global mobile communication booms in modern era, especially in developed countries and economy quickly rising developing countries. At present, different type of smart phones occupy high-end-markets for selling products and stocks to consumers. Markets spread all over the world always have huge capacity and centrality of too many versions of smarts phones. However, national smart phones producers could only obtain market stock but no any profits. So, how does a normal national smart phones producer become attractive or successfully show their power is a serious problem we have to address, also urgently. Nokia is a good example to illustrate how company got success on sold out their products and earned many profits. At first, smart phones were invented by relative experts yet. Instead, Nokia is famous for its first version of functional phone in last 10 years ago. The cool cover type phone design and advanced inner software or Symbrain system have won most people's heart worldwide, but the most successful feature Nokia's functional phone can bring to us is distant communication. A phone call is convenient enough to transfer message rather than writing letters or transport to the place to have talk. However, the invention and notion of internet and online surfing using wifi during the later days are in people's mind, functional phone for just calling cannot fulfil people's colorful lives. Also, other producing smart phone companies like Apple and Microsoft came out with unstoppable power and supports at that time, which restrain the development of Nokia's function phones and gradually control the most market share in the whole world. Therefore, to save Nokia's reputation, Microsoft made the surprising announcement that it was purchasing Finnish mobile handset maker Nokia for $\$ 7.2$ billion, a merger aimed at building Microsoft's mobile and smartphone offerings. It is said that Microsoft is well-known for its software program on laptop for most times. But this time, the thing Microsoft made a decisive call to merge Nokia, an outdated functional phone maker, is impressive enough for people to think over reason behind. Nonetheless, the background information of nowadays clearly shows how people want to use phones more personally and user-friendly. Obviously, Microsoft knew that specializing software exploitation cannot make such massive returns for the whole corporation and employees. Merging Nokia not only makes them receive higher customer's basement reputation but also enriches operating department, although there are some problems needed to solve: the merger faced even more complexity after the ink dried on the contract-namely, the challenges of integrating employees from different cultures. 
International business negotiation case study such as this one underscore the difficulties that companies face when attempting to negotiate two different identities.

Our research topic is 'Analysis of The Deal Between Microsoft And Nokia.'. Admittedly, this topic perfectly connects technological cap between several years ago and modern times. Even though, the case of merging happened in 2013, too far from now, reviewing this classical event influenced on economy, business, financial and entrepreneurship is a good source for new learner to quickly realize negotiating models and how it affects civilians and specialized course. Also, our research is quite easy understanding and comprehensive from different aspects. The most necessary reason we chose this topic is that people might learn same lessons happened on current year and prepare how to deal with similar economic fallacy or embarrassed situation later on. People are gradually evolved into more intelligent species; they have the ability to judge the past from the present. For example, people in last century encountered bubble economy - the most famous financial crisis, tulips boom and bust. So, later generations will be taught to come up with more rational emotion to prevent such crisis again. Our case has the same function as mirror to correct past and manage future. By the way, the subject has not been touched by many researchers, so we did.

In our research paper, the start-up business structure and its ethical core of both Microsoft and Nokia were analyzed in following paragraphs. Since we had the topic related to negotiation, long and detail information in their merge negotiation are discussed from distinct aspects: respiration price, bargaining zone, aspiration level and negotiating skills or BATNA. Undoubtedly, all explanation and evidences of academic terms show our precise on what is really going on in this case. What are both sides really offer is the main question we discussed in our body paragraphs. In addition, several marketing analysis model, such as Porter's 5 forces and SWOT, are used to estimate pros and cons for both sides in this negotiation. Speaking on model we used, one of the most important reasons is that it could help researchers and devisors to find out their best option on business administration. Also, the two methods are so famous that many people confirmed their credibility. Plus, it is really pivotal to claim that we considered current situation, COVID-19, as factor to influence negotiating result. How the merged company affected us differently from 2013, the first year they got merge. Eventually, a reflection of this case has written by listing high-light point in negotiation, and a suitable suggestion for future technological corporation development under big varied context. We believed our paper concluded unique analyzing process and results due to comparing different situation, and how people react to them.

Last but not the least, all hypothesis we sketched served for a unified objective, what do readers fully respond to this paper, whether they will agree with our arguments. In another words, many ways to consider making a right scheme for company to execute are introduced by so many descriptive words in every specific section we created. And we want readers to involve with that, and be cultivated to think like a real rational economist. Taking almost every circumstance may happen seriously in whatever negotiation or daily lives, and be appropriated to manage all limited resource in future use. Just like what we said in our paper, Microsoft has already behaved like a wise economist. Bill Gates, the prior CEO of Microsoft, directs the company a hopeful future development, and obtain both applause and money.

\section{The Introduction, Business Model and weakness of Microsoft}

Microsoft is an international company of technology which mainly focuses on the development of software and operational system of PC. It is known for its Windows system, and the series of Office software. Microsoft was founded in 4th, April, 1975 by Bill Gates and Paul Allen, and the head quarter is located at Redmond, State of Washington. It's now the greatest for producing computer software, and the development of software in personal computers. On 23th, June, 2021, Microsoft's market value exceeded 2 trillion dollars, becoming the second enterprise in the United States after Apple to do so. 
Microsoft is primarily an Office and Dynamics business (ERP, CRM systems) and acquired LinkedIn business. The essential business is Office. The Office 365 product is a subscription model that charges an annual fee, allowing free use of all Office applications on multiple platforms, and providing free updates. Office subscription business is divided into enterprise form and individual form. The subscription business model was a huge success, progressed from selling software to providing office SaaS services. Unlike a one-off payment, a subscription system provides a constant requirement of subscription revenue each year, allowing users to keep up to date and reduce their extra cost. Microsoft was primarily focusing on a server product and cloud service (Azure, Windows Server, Visual Studio) and Enterprise Service (Consulting Service, Support Service). The essential part is the Azure enterprise cloud service. Microsoft Azure has the world's greatest market involved in, and as an IaaS service, it requires a lot of capital. For example, the market involving Microsoft. Azure in China is markedly noticed. The Microsoft Intelligence Cloud also offers a range of PaaS services. Microsoft operates a mixed cloud business through Azure Stack. In recent years, with Google, Apple, Samsung, etc. to implement the operating system free strategy, Microsoft's disadvantage in the mobile becomes increasingly apparent. According to the data, Microsoft had only $3.0 \%$ of the mobile operating system market in 2014, while other systems like Apple IOS, and Android OS possess each $10.0 \%$ and 44\%. The status of windows Phone was non-ideal in 2014. Later, Microsoft offered the section of the initiative free, and Qihoo 360, millet, Tencent and other Chinese Internet companies to upgrade free of using Win 10.

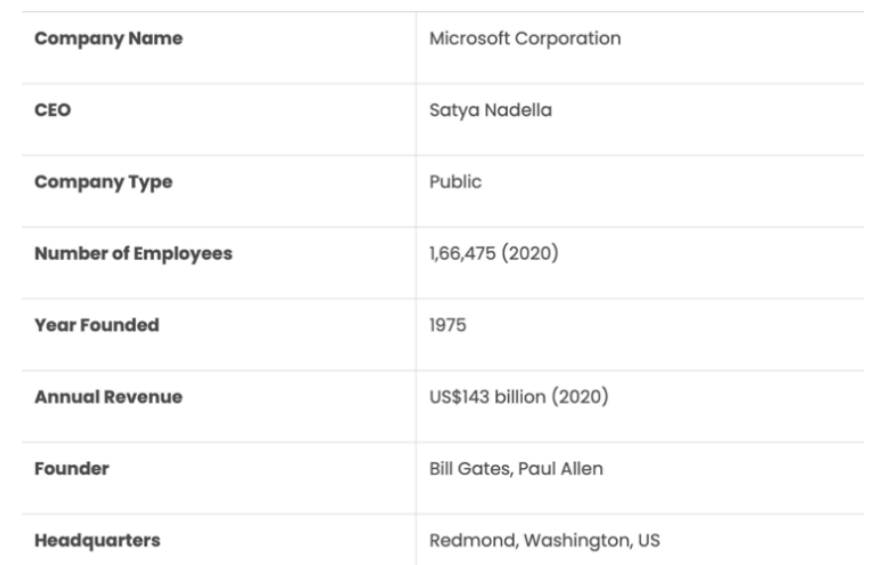

Fig 1. General introduction of Microsoft.

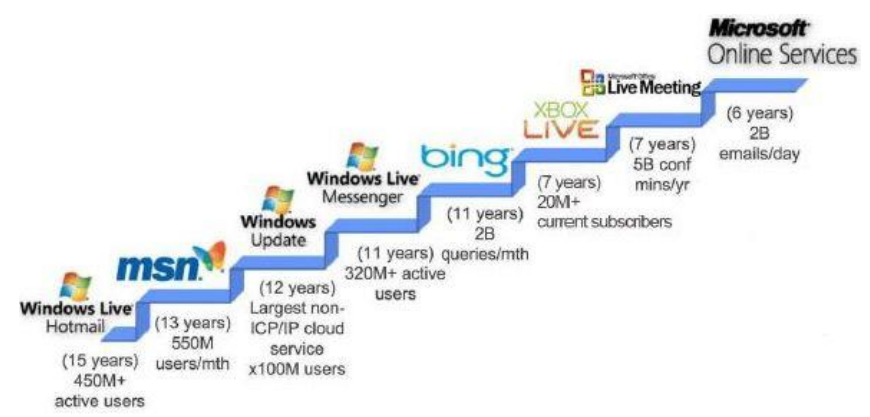

Fig 2. The developing process of Microsoft. 


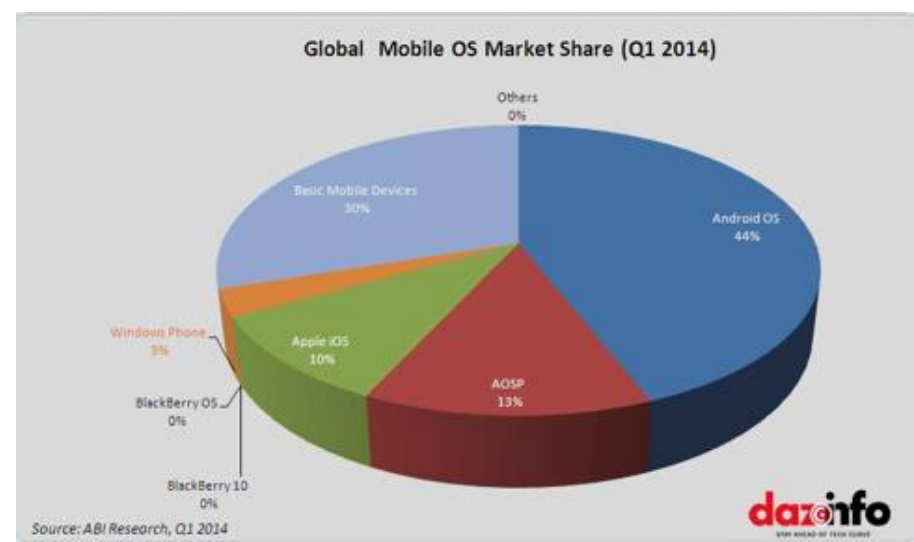

Fig 3. Mobile program market share in 2014

\section{The Introduction, Business Model and weakness of Nokia}

Nokia Corporation is an international company of mobile communication which manly focuses on the production of related facilities and services. It is founded in 1865 for lumbering soon focusing on the development of mobile phones. The head quarter of Nokia Corporation is located at Espoo, Finland. There are three broad categories of Nokia handsets, which are divided into smart and nonsmart phones and tablets. Its smart phones use Symbian, Meego, Windows Phone, and Asha systems, while non-smart phones use the Symbian S30 or S40 systems. Tablets and Windows Phone smart phones are part of the Lumia family of devices that carry Windows RT systems. On 18th, October, 2012, Nokia lost 969 million euros for three seasons, mainly focusing on the great decline of the sale volume of its smart phone, and all the consumers were waiting for the next generation of next WP system. At that time, thr Lumia family devices could only carry the Windows phone 7.5 system, but could not develop to the Windows phone 8 system. Indeed, two other systems which are Android and Apple IOS occupied too much market share which created an unfriendly market for the market of products of Nokia. The market and the development created such a great loss to Nokia.

The enterprise culture of Microsoft is closely related to its founder, Bill Gates. He is innovative and decisive while making decisions according to the situations and developing the technological devices of Microsoft. One of the typical examples is that abandoning the program of creating smart phones and its operating system which wasn't an effective product comparing to other companies. It was the program that Microsoft focused the most. The decisiveness of Bill Gates made a constant investing on a plan which was seemingly failed ceased, and Microsoft avoided losing capital from that program which will eventually go failed. Even though Bill Gates is no longer managing the Microsoft, his overall contribution to the Microsoft is great.

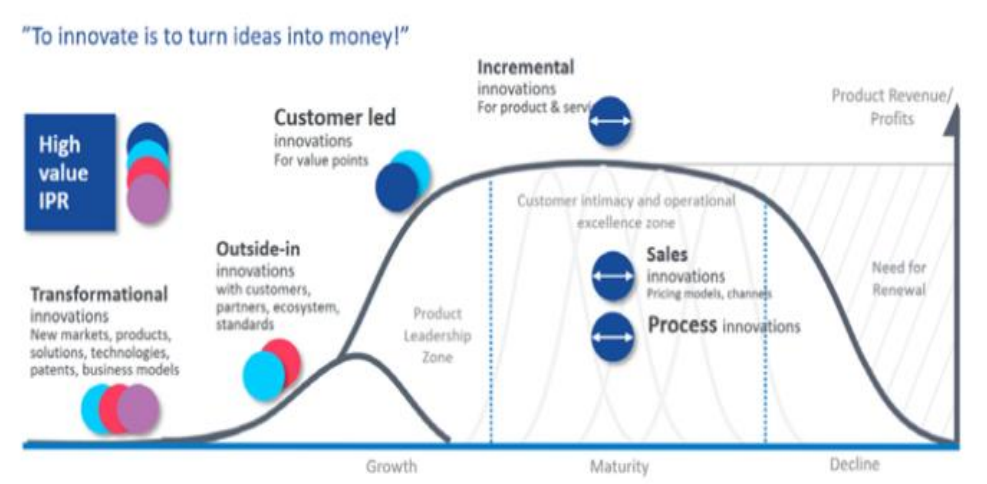

Fig 4. Nokia's innovation management strategy (based on Geoffrey Moore's "Crossing the Chasm", 1993); the goal of the strategy is to cross the chasm faster. 


\section{Case study: Microsoft merged Nokia deal making}

\subsection{Microsoft and Nokia negotiating Reservation Price}

On September 3, 2013, Steve Ballmer (current CEO of Microsoft) and Stephen Elop (former CEO of Nokia) issued an open letter that Microsoft intends to acquire Nokia's equipment and services business. In regular transactions, Nokia's closing price was US\$3.85-about 50\% higher than the same period last year, but far below the historical high, with a market value of nearly US $\$ 14.3$ billion. Unfortunately, the evaluation price and reservation price for Nokia is apparently low: When Elop first joined Nokia, Nokia was valued at 29.5 billion euros, compared with 11.1 billion euros when Microsoft announced the acquisition. (Tencent News \& TMTPOST provided)

On the other hand, can we say that the difference between 29.5 billion euros and 11.1 billion euros is what Microsoft costed for investment on Elop for later brought Nokia back. It's worth though on some extent that Microsoft and Nokia eventually reached a good deal for inventing new functional tech to people who advocate this deal making.

\subsection{Microsoft and Nokia negotiating Bargaining zone}

On the evening of September 2, 2013, Microsoft announced that it would acquire most of Nokia's mobile phone business for 3.79 billion euros (about 5 billion US dollars), and another 1.65 billion euros (about 2.18 billion US dollars) for the purchase. The total price of Nokia's patent license is approximately 5.44 billion euros (about 7.17 billion US dollars) due to this transaction. In addition, Microsoft obtained the special right from Nokia's technology. In other word, Nokia will sell a 10year non-exclusive patent license to Microsoft.

From the employee's perspective, the management of new employees in Microsoft is another issue they made decision when negotiated. According to the report, Nokia's net layoffs reached 11044 population, making Nokia's average number of employees in 2020 to 92,039 population, which means that the total number of employees has fallen by $11 \%$ in just two years. Regional analysis shows that employment figures all over the world have declined, but China has experienced the largest decline. Last year, the number of employees in China fell by $12 \%$ to 13,749 population, a decrease of nearly 3,500 population since 2018 . However, finding ways to recruit intellects in Microsoft is another condition they made in deal. The new constitution of Microsoft is trying to improve this situation by letting 32,000 employees originally belonged to Nokia joining Microsoft, including 4,700 Finnish employees and approximately 18,300 employees in the manufacturing department. They are all concluded into bargaining zone in this deal. (TMTPOST provided data)

\subsection{Microsoft and Nokia negotiating Aspiration level}

Prior to the acquisition, the former CEO of Nokia, Stephen Elop is confident to their products, and said 'Nokia is not only a hardware developer, but also a software and service development about cloud computing platform.' He denied that Nokia will be purchased by Microsoft in the future and reclaimed that Nokia will operate as an independent company. When talking about how the Nokia Windows smart phone is different from the similar products of Samsung and HTC, Elop said that Nokia has gathered the company's resource advantages and invested in the Windows smart phone strategy, which has obvious advantages. Therefore, although the Windows smart phone of all manufacturers are interoperable, some services and functions are unique to Nokia, plus Nokia's Hardware advantage, this is the unique competitiveness of Nokia Windows Phone. Therefore, Nokia had a very high level of aspiration price about themselves, about 29.5 billion euros, which is also Nokia's dream price if it sells to others.

\subsection{Negotiation skills and BATNA in acquisitions}

For the acquisition, we must understand the background of the other party. First of all, let's get to know Nokia. Nokia has failed in the past two years, and many people have lost support for Nokia. Even Nokia was all the rage in the small town of Shanghai, but also closed its doors, not selling the 
top ten chocolates! It's not Nokia's style. The original Nokia has one basic manpower, which is on the same level as Apple. But now Nokia is beginning to change its assets and is considering relocating its headquarters in Finland in order to be able to continue operations. So overall it is still a downward trend. Then there is Microsoft. For Microsoft, Microsoft is now moving from "behind the stage" to "behind the scenes". It is now to provide the platform and commercial digital computing when it was at the peak of Microsoft, and it was very capable on servers, development tools and Windows, far away from the computer, those essential or former strengths of Microsoft, are now gone. For example, the Internet, as well as the perception of the consumer market, are fading away. Therefore, this acquisition is undoubtedly beneficial and harmless, like two sleeping giants working together. Just like the cooperation between Disney and Pixar, it will only bring better film and television works as well as product promotion and peripheral products. Then the first favorable factor in the negotiations between Microsoft and Nokia is that Microsoft can use cash from overseas branches to pay for the acquisition, because Microsoft has about $\$ 66$ billion in cash in overseas branches. The second favorable factor is that Nokia is still an independent operating company, but through acquisitions, the company's structural profitability has been shifted from mobile phones to software services and network hardware solutions. Nokia's existence value lies in its patents in mobile phones, as well as its long-term hardware advantages. It has good and rich experience and resources in the management and quality of the global supply chain and hardware after-sales service. Therefore, Microsoft is now very much in need of a future mobile phone manufacturer to explore its various goals in the mobile field and is determined to conduct better product adaptation through cooperation with local or foreign manufacturers, so as to guide more manufacturers to join the Microsoft camp. Take Intel, for example. Microsoft and Intel have joined forces to develop a brand-new processor with a performance increase of ten thousand times. Microsoft and Intel alone have made millions. After all, Nokia can be regarded as the overlord of the original mobile phone industry. It is very knowledgeable and experienced in the development of software and hardware. Therefore, their negotiations can start with Microsoft's ability to agree and provide Nokia's software development. It is no longer the simple Symbian system before, and Microsoft can help upgrade and upgrade. The second is that it can solve the problem of Nokia's layoffs and give employees better treatment. Employees do not have to be scared every day, afraid that they will be fired tomorrow. Finally, there is Nokia. Nokia can provide Microsoft with a big platform. After all, this is what Microsoft wants most. And Nokia can provide Microsoft with its most valuable things, which are operators and channels. First of all, operators refer to providers that provide network services. For example, manufacturers of communication equipment such as Huawei, ZTE, Nokia, and Ericsson are called manufacturers, while companies such as China Mobile, China Unicom, China Telecom, and China Radio and Television are called operators. So Nokia, as a supplier larger than the operator, is very advantageous because of its wider channels. As Nokia's CEO Long Peikai said: "We are a trusted partner in key network areas. Whether it's delivering operator-level networks or cooperating with the world's most demanding Internet companies, we have a wealth of Experience. We have strong capabilities in key network core technologies, such as open radio access networks and virtual radio access networks, and we are working hard to launch a $100 \%$ cloud-native software product portfolio." The Nokia, the key network market for communication service providers and Internet companies and industry enterprises, is also expanding. The last is BATNA, if Microsoft cannot upgrade the Symbian system, or must pay a license to upgrade the software. Then Nokia can also reduce its large platform or operating channels a bit smaller, or not only develop platforms for Windows.

\subsection{Post-acquisition business strategy}

Go over the financial channel development of Microsoft, the successful company on base of exploiting software and feasible resource management. For Microsoft, it has same process tendency. At the beginning of Microsoft, in 1975, 19-year-old Bill Gates dropped out of Harvard University and founded Microsoft with his high school alumnus Paul Allen, focusing on selling BASIC interpreters. Unfortunately, they tried to enter the home computer market by designing the MSX home 
computer standard, but the result was not very successful. This failure caused Microsoft lost many moneys, however, it also meant the introduction step began. For clear statement, Microsoft now need angle investment from relatives or friends. They only got their idea of making computer program with any specific expression. So, finding investment became the first problem toward Bill and Paul. After overcoming this difficulty, Microsoft can be described as smooth sailing, and the upward period is obvious. Not only they signed contract with IBM and sold for over 100,000,000 dollars that year, but successfully release Microsoft Windows 1.0, which is the first product in the Windows series, and it is also the first attempt by Microsoft to implement a graphical user interface on a personal computer operating platform. Obviously, Microsoft in 1985 reached in growth step. Congratulations! To be honestly, the over-area from growth to maturity for Microsoft contained apparent and unified behavior, which is to acquire other companies for extending self-scale and erecting their own company brand in the world. Therefore, it is important for Microsoft to accumulate public credibility or consumer basements. So, among the many acquired company, Nokia had a wise negotiation with Microsoft, for one thing, it saves the enterprise culture of traditional making phone's manufacture under the circumstance of modern change and personalization; for another thing, many employees originally from Nokia had been kept, which means they still worth the piece of salary Microsoft gave. Admittedly, the join of Nokia to Microsoft at the stage of burgeoning is beneficial for both sides, but especially for Microsoft as letting tiger with wings, as old Chinese saying.

From the perspective of two useful models: Porter's five forces and SWOT marketing analysis, Microsoft also carry itself with ease. Porter's five forces includes: power of supply, power of buyer, power of potential opponents entering market, power of substitute and the power of market itself. Obviously, Microsoft is focused on exploiting safe and variable office software, which have some powerful competitors, for word dox., office is the competitor of WPS. Therefore, it is available for Microsoft to enhance their ability on producing more goods, and persuading people to become their potential consumers. The reason is Microsoft owned a strong leverage on software entrepreneurship, so other small companies have no enough competence comparing with Microsoft, and might gave up depriving potential customers of Microsoft. In short, Microsoft will use their advantages on suppliers and buyers to overcome brand shortage on too many substitutes.

Nokia hold different state:

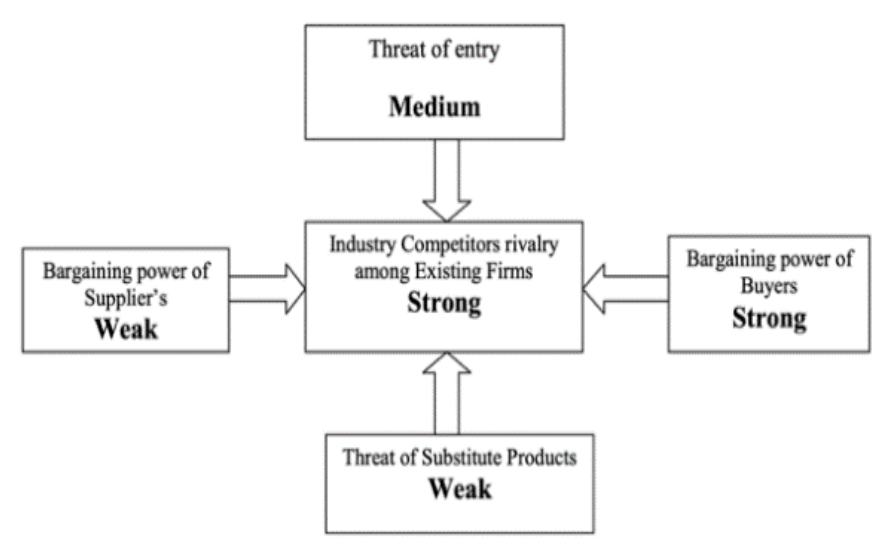

Fig 5. Porter's five forces analysis on current Nokia

When speak on SWOT analysis, results appear similarly. SWOT are the acronym of 'Strength' 'Weakness' 'Opportunity' and 'Threat'. CEOs always combine four characteristics into a matrix, which helps them to locate where is their company stands, and maximize the strength with opportunities, or avoid the weakness with threats. It seems that merging Nokia is a good opportunity for Microsoft to expand its authority in market share. Plus, Microsoft have already got accumulated reputations, which is counted as their strength. Therefore, win-win situation occurs. 


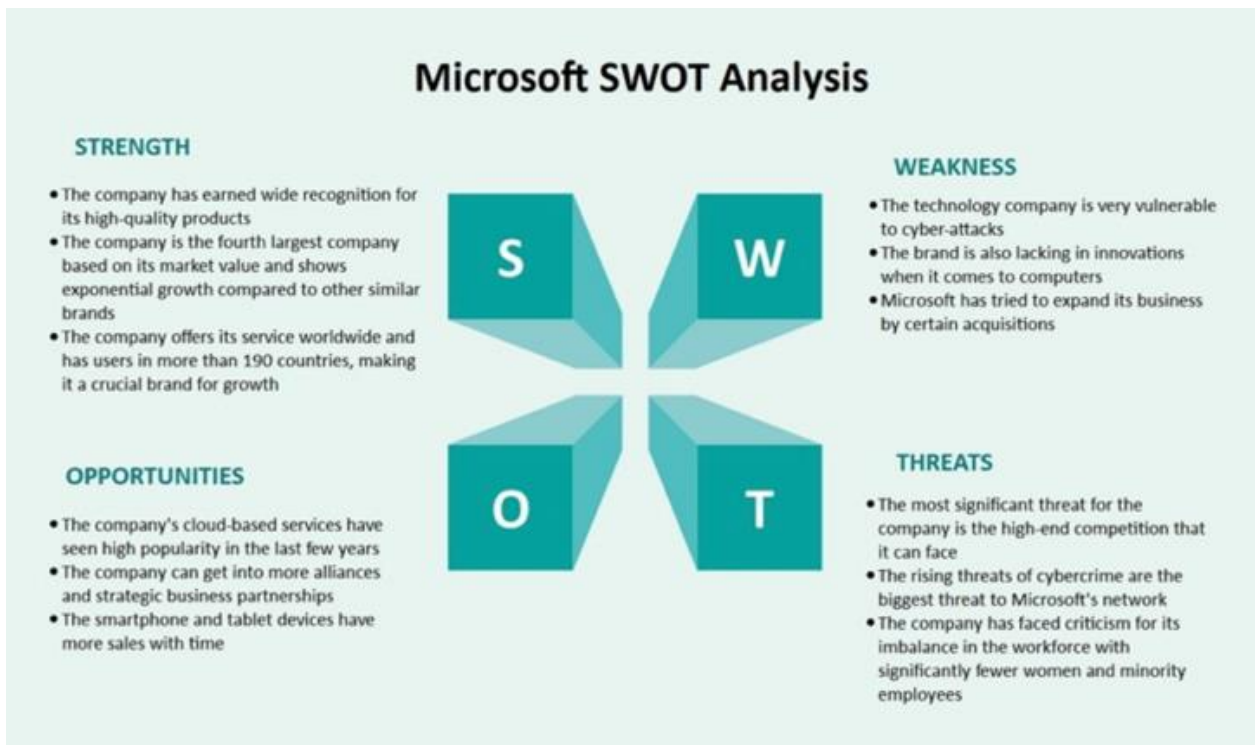

Fig 6. Microsoft SWOT analysis, resource from EdrawMax Online

Here are some reasons for the joined forces:

First off, the post-acquisition strategy is that Nokia and Windows Phone focus on a "peopleoriented" user experience, which integrates many services of Facebook and Twitter. Nokia will bring diversified functions to these terminals, such as navigation and maps, so it becomes a unique product that does its best to satisfy users. Therefore, the original ideas cannot be lost, and new ideas can be added.

Then, there is also no limitation to software development, such as software development only. Both parties can try to make new products, such as using Microsoft's system and Nokia's technology to develop tablets or computers.

Last but not the least, there are also in-depth exchanges between the employees of both parties, which can better help the company develop products from maximizing the use of human resources.

\section{Limitation}

This research has three limitation on designing the structure. First, all numbers in reservation price and aspiration level sections are found in related news and record made by Microsoft. Therefore, we definitely do not own the ability to observe the real value on this deal making. (ability to observe is stand for A in RAVEN method on analyzing evidence we gave in article) Second, the quantitative data about the true approval from people who use Nokia and the combination of Nokia and Microsoft is actually small. For researching process, we only located on our friends and family members, and tried to search more information online. It is not enough for making such a big poll on deciding whether this deal making offer people happier and convenient lives later on. Last but not the least, this deal making has last for several years and nowadays people's mind on using smart phone and demand on 5G cannot fit that circumstance anymore, which cause even unfair altitude to the development of Nokia's functional phones. The spontaneous compare and contrast on current standard of phone usage will lead to our misjudge on writing this article. Therefore, it is better for us to find old record and news report on the deal.

\section{Conclusions}

After Nokia was assigned to Microsoft, the original engineers participated in the development of Windows Phone. According to the Nokia's exclusive API that opened after the Nokia Windows Phone GDR2+Amber update and the release of Lumia 1020, Nokia prefers an open model and is more user-friendly. Introduce more "grounding gas" functions, such as double-tap to wake up the 
screen, always overview. This is conducive to the development of Windows Phone in the direction of "More Human" in the future. Nokia has changed its slogan "Technology People-oriented" to "More Human". On the other hand, Microsoft still upholds this kind of arrogant Windows XP version for the promotion of Win8. As for the abnormal usage habits and product experience, it seems that the users are "own business"-the conceptual closure is destined for users to go away. The farther. Going further, Apple's App Store, iTunes, and iBooks are infiltrating all corners of the industry with their own hardware terminals. Amazon has already established an e-commerce + cloud service + Kindle system, while Microsoft is still Windows. In a large ecosystem, the inheritance of user experience is a more terrifying product. After all, the perfect experience brought by iCloud and Chrome has made users no longer willing to pay a high cost to learn Microsoft's set of products that can never be learned. Logically, it is foreseeable that users will become more and more trapped in the arms of Amazon, Apple or Google. As for Microsoft, it is no longer in the world.

\section{Reference}

[1] A with Honors Projects in Parkland College, Market Strategy from Microsoft Acquiring Nokia. (Weiting Li (pinyin), 2013)

[2] Baidu Library, Research on business model and application based on value chain thinking. (ZANGWEI \& CAOQIANG (pinyin), 2011)

[3] Baidu academy, Microsoft free and digging into pain points. (CHENRUN (pinyin), 2015)

[4] Biz fluent business website, Introduction to Microsoft Corporation. (D. Faith, 2017)

[5] ETLA - The Research Institute of the Finnish Economy, Microsoft Acquires Nokia: Implications for the Two Companies and Finland. (Ali-Yrkkö, Kalm, Pajarinen, Rouvinen, Seppälä, Tahvanainen, 2013)

[6] HEIMA Website-let entrepreneur not be lonely, how far Microsoft can go after it buys Nokia. (WUSHUXIANSHENG (pinyin), 2013)

[7] JIEMIAN GLOBAL NEWs, what power does Microsoft have to acquire Nokia. (ZIJINSHAN (pinyin) technology, 2020)

[8] WANGYI (pinyin) technology, Nokia announces changes to its operating model and leadership team. (MAOXINEN (pinyin), 2020)

[9] ZHIHU public discussion website, \#several reasons to explain why Nokia has been acquired\#. (Tato, 2012; Ender, 2018)

[10] ZHIHU public discussion website, \#the commercial mode of Microsoft\#. (mike world, 2021)

[11] HYPE (2007) Nokia Building an innovation ecosystem

[12] https://www.hypeinnovation.com/hubfs/content/case-studies/nokia-case-study-en.pdf 